\title{
Restrictions du droit de grève en Australie
}

\section{Shae McCrystal}

\section{(2) OpenEdition \\ Journals}

Édition électronique

URL : https://journals.openedition.org/rdctss/2048

DOI : $10.4000 /$ rdctss.2048

ISSN : 2262-9815

\section{Éditeur}

Centre de droit comparé du travail et de la sécurité sociale

\section{Édition imprimée}

Date de publication : 1 avril 2018

Pagination : 140-143

ISSN : 2117-4350

\section{Référence électronique}

Shae McCrystal, « Restrictions du droit de grève en Australie », Revue de droit comparé du travail et de la sécurité sociale [En ligne], 1 | 2018, mis en ligne le 01 novembre 2021, consulté le 12 novembre 2021. URL : http://journals.openedition.org/rdctss/2048; DOI : https://doi.org/10.4000/rdctss.2048

\section{(c) (i) (9)}

Revue de droit comparé du travail et de la sécurité sociale est mise à disposition selon les termes de la Licence Creative Commons Attribution - Pas d'Utilisation Commerciale - Pas de Modification 4.0 International. 


\section{SHAE MCCRYSTAL}

UNIVERSITÉ DE SYDNEY

\section{RESTRICTIONS DU DROIT DE GRÈVE EN AUSTRALIE}

Deux décisions récentes en Australie ont restreint l'accès au droit de grève des salariés australiens. Nous examinerons ci-dessous d'abord le contexte juridique du droit de grève en Australie puis les évolutions récentes de ce même droit pour mettre en évidence les restrictions dont il fait l'objet.

\section{I - LES ACTIONS COLLECTIVES PROTÉGÉES}

En Australie, la capacité des salariés à déclencher légalement une grève est régie par la partie 3-3 du Fair Work Act 2009 (Cth) (FW Act). Au cours des négociations sur les accords d'entreprise, les salariés et leurs agents négociateurs peuvent engager une action collective ou un moyen de pression (industrial action) protégé à l'appui de leurs revendications relatives au projet d'accord collectif. Une fois que les salariés ont entrepris une telle action, les employeurs peuvent y répondre en recourant à leur propre action juridiquement protégée sous la forme d'un lock-out des salariés. Un moyen de pression protégé est une action qui remplit les critères de la " grève » telle que définie à l'article 19 du FW Act et qui satisfait aux conditions légales requises pour obtenir le statut d'action "protégée ». Les conditions préalables légales pour qu'une action soit protégée sont entre autres, les suivantes:

- L'agent négociateur a obtenu de la Commission du travail équitable (Fair Work Commission - FWC) le droit d'organiser un vote (article 443 du FW Act), et l'action proposée a été approuvée lors d'un vote des salariés concernés (article 409 (2) du FW Act)1,

- L'action est organisée et engagée pour soutenir ou faire avancer les réclamations relatives à l'accord proposé (article 409 (1) du FW Act),

- L'agent négociateur tente sincèrement de conclure une entente avec le chef d'entreprise concerné (article 413 (3) du FW Act),

- Le préavis de grève obligatoire (articles 413 (4) et 414 du FW Act),

- L'agent négociateur n'a « enfreint aucune ordonnance le visant à propos de l'accord collectif, ou d'une action collective liée à l'accord collectif, ou d'une question soulevée au cours de la négociation de l'accord » (article 413 (5) du FW Act),

- L'accord est arrivé à sa « date d'expiration » (article 413(6) du FW Act), et

- La FWC n'a pas autrement suspendu ou mis fin à une action revendicative liée aux négociations en cours (articles 423-426 du FW Act).

Lorsque l'action collective protégée est conforme aux conditions préalables susmentionnées et est engagée dans les 30 jours suivant le vote approuvant l'action, elle peut se poursuivre indéfiniment, sauf si une entente survient, ou si la FWC ordonne que l'action soit suspendue ou stoppée en raison de son impact sur les parties à la négociation ou sur des tiers.

1 Concernant l'obligation de vote, voir B. Creighton, C. Denvir et S. McCrystal, "Strike Ballots and the Law in Australia", Australian Journal of Labour Law, 2016, Vol. 29, n² 2, p. 121. 
Une action qui n'est pas protégée (non conforme aux conditions légales) peut faire l'objet d'une injonction ou de dommages-intérêts en Common law pour toute une série de motifs, dont la rupture de contrat ou la réalisation d'une infraction économique ${ }^{2}$. En outre, une action qui n'est pas protégée peut faire l'objet d'une ordonnance par la juridiction du travail, la FWC, de cesser, de ne pas organiser ou d'éviter que soit menée un moyen de pression en vertu de l'article 418 du FW Act. Si une telle ordonnance est violée, une injonction ou une sanction civile peut être obtenue auprès de la Cour fédérale d'Australie.

Comme on peut le voir, mener un moyen de pression en toute légalité en vertu du FW Act implique pour les négociateurs de l'accord collectif de bien maîtriser la complexité des conditions préalables. Deux décisions récentes en Australie ont renforcé les difficultés en vue de s'engager dans une action légale et ont facilité les possibilités de remettre en cause la légalité de l'action collective.

\section{II - ACCÈS AU DROIT DE GRÈVE}

L'une des conditions préalables aux actions revendicatives protégées figure à l'article 413 (5) du FW Act, selon lequel l'agent négociateur concerné n'a "enfreint aucune ordonnance le visant à propos de l'accord collectif, ou d'un moyen de pression lié à l'accord collectif, ou d'une question soulevée au cours de la négociation de l'accord ». Le sens de cette section a été récemment examiné par la Haute Cour (High Court) dans l'affaire Esso Australia Pty Ltd $v$ The Australian Workers Union (Esso) ${ }^{3}$.

Au cours des négociations en vue d'obtenir un accord d'entreprise, l'Australian Workers Union (AWU) a mené une action revendicative. L'employeur, Esso Australia, a obtenu une ordonnance en vertu de l'article 418 du FW Act, enjoignant à l'AWU de cesser l'organisation de l'action collective non protégée. Croyant à tort que l'action collective qu'elle organisait était protégée, l'AWU l'a poursuivie. Toutefois, le caractère non protégé de ce moyen de pression fut jugé ultérieurement, ce qui signifie que l'AWU mena, pendant une courte période, des actions collectives en violation de l'ordonnance de la FWC.

L'employeur demanda par la suite à la Cour fédérale d`Australie de déclarer que l'article 413 (5) du FW Act avait pour conséquence d'empêcher le syndicat d'entreprendre tout moyen de pression protégé dans le cadre des négociations relatives à l'accord en cause dans la mesure où l'AWU avait préalablement enfreint l'ordonnance de cesser le précédent moyen de pression non protégé.

Conformément à l'interprétation précédente de l'article, les tribunaux inférieurs statuaient que l'article 413 (5) n'empêchait nullement un moyen de pression protégé lorsque la violation d'une telle ordonnance avait été rectifiée ou que l'ordonnance était expirée ${ }^{4}$. Cela signifiait que si le syndicat remédiait à son infraction, ou n'était pas en infraction au moment où il cherchait à entreprendre une autre action collective, il pouvait entreprendre un moyen de pression protégé.

Cette approche a été rejetée en appel par la Haute Cour dans l'affaire Esso, avec une majorité de 4 contre 1. Considérant le contexte légal et l'historique de la disposition, il a

2 Voir A. Stewart et al, Creighton and Stewart's Labour Law, 6 $6^{\text {th }}$ ed, Federation Press, 2016, ch. 26.

3 [2017] HCA 54.

4 Esso v AWU (2015) 253 IR 304, Esso v AWU (2016) 245 FCR 39. 
été conclu qu'un agent négociateur ne pouvait pas remplir les conditions préalables à un moyen de pression protégé si, à un moment quelconque des négociations de l'accord, il avait enfreint une ordonnance de la FWC ou d'un tribunal. Considérant le fait que le droit d'entreprendre un moyen de pression protégé constitue un "privilège ", la Haute Cour estime que le but apparent de la disposition était «d'assurer que les personnes qui ont démontré qu'on ne pouvait pas leur faire confiance quant au respect d'une ordonnance concernant l'accord ou des questions relatives à la négociation dudit accord ne doivent pas pouvoir bénéficier de l'immunité accordée dans le cadre d'un moyen de pression protégé ${ }^{\text {». }}$

Ce jugement exclut effectivement tout moyen de pression protégé pour les salariés lorsque leur agent négociateur a enfreint une ordonnance de la FWC ou d'un tribunal pendant la négociation de cet accord, indépendamment des circonstances entourant la violation ou lorsque la violation est mineure, involontaire ou simplement technique. Tout moyen de pression légal est alors impossible jusqu'à la négociation ultérieure d'un autre accord.

Le régime du FW Act confère à la FWC le pouvoir de recourir à diverses mesures relatives à la négociation d'un accord, y compris des ordonnances de négocier de bonne foi et des ordonnances relatives à des actions collectives, afin de faciliter la conclusion équitable, efficace et flexible d'une entente. Les ordonnances font partie de l'esprit de la Loi, et les violations involontaires (ou non) sont courantes. Exiger le strict respect de toutes les décisions, sous peine de perdre l'accès à un moyen de pression protégé, a pour effet de judiciariser davantage un système qui devrait être suffisamment souple afin de s'adapter au flux et reflux naturel des conflits du travail qui sont l'essence de la négociation collective. Cela impose une restriction de l'accès au droit de grève qui constitue une "punition » pour les transgressions passées, aussi mineures soient-elles. En désaccord avec les juges majoritaires de la Haute Cour, le juge Gageler a fait observer, dans son opinion dissidente, que cette approche créait des hors-la-loi sociaux et n'était pas conforme à un régime juridique conçu pour être « équitable, souple et efficace ${ }^{6}$ ».

\section{III - RESTRICTIONS DES ACTIONS DE GRÈVE}

La deuxième décision ayant des implications profondes pour l'accès au droit de grève en Australie est celle relative à l'affaire Sydney Trains ${ }^{7}$.Sydney Trains est la société gestionnaire du réseau de trains dans la banlieue de Sydney. Le Syndicat australien des chemins de fer, des tramways et des bus (The Australian Rail, Tram \& Bus Industry Union $R T B U$ ) représente les salariés de Sydney Trains. Au cours de négociations prolongées en vue d'un nouvel accord d'entreprise, le RTBU a notifié la tenue d'un moyen de pression protégé sous la forme d'un refus d'exécuter du travail en temps supplémentaire pour une période indéfinie et d'un arrêt de travail de 24 heures. Toutefois, avant que ce moyen de pression ne commence, Sydney Trains saisissait la FWC en vue d'en obtenir sa suspension.

L'article 424 du FW Act prévoit en effet que la FWC doit suspendre ou mettre fin à tout moyen de pression protégé qui menace de mettre en danger la vie, la sécurité personnelle ou le bien-être de la population ou d'une partie de celle-ci ou de causer des

\footnotetext{
5 [2017] HCA 54 [53].

6 [2017] HCA 54 [103], [104].

7 [2018] FWC 632.
} 
dommages importants à l'économie australienne ou à une partie de celle-ci. Lorsque l'action est suspendue, elle peut reprendre après la suspension. Si elle est annulée, les parties entament alors une période de négociation post-clôture. Si elles ne parviennent pas à s'entendre sur les questions en suspens, la FWC peut arbitrer les questions qui restent en litige. Sydney Trains et le ministre ont fait valoir que l'action collective projetée mettrait en danger le bien-être des banlieusards faisant la navette pour venir à Sydney et causerait des dommages à l'économie locale.

La FWC a suspendu l'action collective pendant six semaines, estimant que le bien-être d'une partie de la population était menacé en raison du grand nombre de banlieusards faisant la navette et qui dépendent du système de trains pour se rendre au travail, à l'école ou pour "s'occuper de leurs affaires" et de la congestion accrue qui se produirait sur les routes $^{8}$. Il y aurait aussi des dommages économiques pour la ville de Sydney, en raison de I'incapacité, pour les banlieusards faisant la navette, de se rendre sur leur lieu de travail'.

Étant donné que l'action collective projetée a été suspendue et non pas annulée, le différend n'a pas été soumis à l'arbitrage. Après six semaines, le RTBU aurait pu, en théorie, organiser de nouveau une action syndicale. Toutefois, étant donné la similitude des circonstances, il était fort probable qu'une autre décision de suspension aurait alors été rendue; toutefois, dans l'optique d'une décision d'annulation (et non de suspension) de la $F W C$, celle-ci aurait alors mené à l'arbitrage de toute question litigieuse restée en suspens.

L'article 424 du FW Act est conçu pour équilibrer, d'un côté, le droit de grève des salariés et, de l'autre, la nécessité de protéger la communauté des préjudices potentiels causés par le recours à la grève. La restriction des grèves qui sont organisées par les travailleurs des secteurs d'activités dont les services sont essentiels (comme les ambulanciers, les travailleurs hospitaliers et les pompiers) est raisonnable compte tenu de l'impact probable de ces grèves sur la santé et la sécurité publiques.

Cependant, l'interprétation de l'expression "mettre en danger le bien-être » dans I'article 424 du FWAct s'étend bien au-delà de ce que l'on considère habituellement comme un service essentiel. Dans une décision antérieure, concernant l'affaire Monash University $v$ $N T E U^{10}$, un moyen de pression protégé mené par des universitaires a été suspendue parce qu'il a été jugé que la non-publication des résultats d'examen mettrait en danger le bienêtre des étudiants de l'université. Ainsi la suspension de l'action pendant deux semaines a permis la communication de tous les résultats d'examen et a de ce fait, complètement miné l'impact de la grève. De même, la décision rendue dans l'affaire Sydney Trains peut effectivement exclure l'accès au droit de grève pour les travailleurs des transports publics lorsque l'action projetée a un impact important sur l'accès aux transports publics. Dans de tels cas, l'exercice du droit de grève a été contraint de céder face aux inconvénients et à l'inquiétude de ceux qui en auraient été affectés. Ce qui ne semble pas établir un équilibre adéquat. Les deux affaires examinées dans cet article illustrent l'idée que, dans le FWAct, le droit de grève n'est pas du tout un droit, mais plutôt un privilège. L'accès à ce privilège est anéanti lorsque les parties transgressent intentionnellement ou non les règles juridiques qui l'encadrent, ou lorsque l'action collective fait ce qu'elle est justement supposée faire, c'est-à-dire avoir un impact.

8 Ibid dans [44].

9 Ibid dans [46].

10 [2013] FWCFB 5982. 